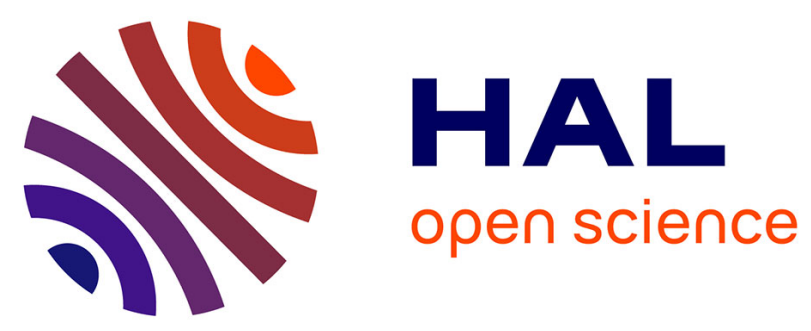

\title{
SDEs with uniform distributions: Peacocks, Conic martingales and mean reverting uniform diffusions
}

\author{
Damiano Brigo, Monique Jeanblanc, Frédéric Vrins
}

\section{To cite this version:}

Damiano Brigo, Monique Jeanblanc, Frédéric Vrins. SDEs with uniform distributions: Peacocks,

Conic martingales and mean reverting uniform diffusions. 2016. hal-01326854

\section{HAL Id: hal-01326854 \\ https://hal.science/hal-01326854}

Preprint submitted on 6 Jun 2016

HAL is a multi-disciplinary open access archive for the deposit and dissemination of scientific research documents, whether they are published or not. The documents may come from teaching and research institutions in France or abroad, or from public or private research centers.
L'archive ouverte pluridisciplinaire HAL, est destinée au dépôt et à la diffusion de documents scientifiques de niveau recherche, publiés ou non, émanant des établissements d'enseignement et de recherche français ou étrangers, des laboratoires publics ou privés. 


\title{
SDEs with uniform distributions: Peacocks, Conic martingales and mean reverting uniform diffusions
}

\author{
Damiano Brigo* $\quad$ Monique Jeanblanc ${ }^{\dagger} \quad$ Frédéric Vrins ${ }^{\ddagger}$ \\ First version: April 4, 2016. This version: June 5, 2016
}

\begin{abstract}
We introduce a way to design Stochastic Differential Equations of diffusion type admitting a unique strong solution distributed as a uniform law with conic timeboundaries. We connect this general result to some special cases that where previously found in the peacock processes literature, and with the square root of time boundary case in particular. We introduce a special case with linear time boundary. We further introduce general mean-reverting diffusion processes having a constant uniform law at all times. This may be used to model random probabilities, random recovery rates or random correlations. We study local time and activity of such processes and verify via an Euler scheme simulation that they have the desired uniform behaviour.
\end{abstract}

Keywords: Uniformly distributed Stochastic Differential Equation, Conic Martingales, Peacock Process, Uniformly distributed Diffusion, Uniform Martingale Diffusions, Mean Reverting Uniform Diffusion, Mean Reverting Uniform SDE, Maximum Entropy Stochastic Recovery Rates, Maximum Entropy Stochastic Correlation, Uniform SDE Simulation.

AMS classification codes: 60H10, 60J60

${ }^{*}$ Department of Mathematics, Mathematical Finance and Stochastic Analysis groups, Imperial College London, damiano.brigo@imperial.ac.uk

†Laboratoire de Mathématiques et Modélisation d'Évry (LaMME), Université d'Évry-Val-d'Essonne, UMR CNRS 8071 monique.jeanblanc@univ-evry.fr

${ }^{\ddagger}$ Louvain School of Management, Université catholique de Louvain, frederic.vrins@uclouvain.be 


\section{Introduction}

We are interested in designing stochastic processes having a uniform law at all times, with a conic type boundary. We focus on diffusion processes. The solution of this problem can be attempted by inverting the forward Kolmogorov or Fokker-Planck equation of the candidate diffusion process by plugging in the desired density, thus deriving the drift and /or diffusion coefficient of the candidate diffusion process that would be consistent with the desired uniform law. This technique was used in the past to construct diffusion processes with densities in exponential families [2,4] and has been used more generally in a variety of contexts in mathematical finance. For example, [7] finds the diffusion coefficient ("local volatility") that is consistent with a probability law extrapolated from a surface of option prices. The paper [6] deals with designing a diffusion process consistent with a mixture of distributions for volatility smile modeling, whereas [5] inverts the Kolmogorov equation to show how two stochastic processes with indistinguishable laws in a time grid under the historical measure can lead to arbitrarily different option prices.

In this paper we initially use a similar technique to guess the diffusion coefficient a martingale diffusion would have in order to model a uniform distribution with conic boundaries. We generalize the approach in [4], adapting it to uniform distributions, as initially done in the preprint [3] in the special case of boundaries that are linear in time. The problem is formulated in Section 2 and a solution is attempted in Section 3 by inverting the Kolmogorov equation. The use of a uniform distribution as candidate solution leads us to use the theory of distributions to complete the inversion. Once the candidate diffusion process has been found, we study it rigorously in Section 4 and prove that the related Stochastic Differential Equation (SDE) admits a unique strong solution. We further prove that the solution has indeed a uniform distribution with the desired conic boundary, and relate our result with the peacocks processes literature [9].

In Section 5 we re-scale the conic diffusion martingale and study the related meanreverting uniform diffusions, where now the uniform law is not conic but constant. Two special cases of interest are standard uniforms and uniforms in $[-1,1]$, which can be used to model for example maximum entropy recovery rates or random probabilities and random correlations, respectively.

In Section 6 we make some specific choices for the conic boundary. The square-root-oftime case coincides with a previous case of peacock process [9]. The linear time boundary case is new, to the best of our knowledge, and provides a new case of peacock process. In this special case we also study the process local time at the boundaries, to find it is zero, and the activity, finding that the pathwise activity of the mean reverting diffusion tends to vanish for large times. We further implement an Euler scheme for the martingale diffusion with linear time boundaries and show that the simulation confirms the uniform behaviour over time.

We conclude in Section 7 by summarizing our findings and by hinting at other different conic boundaries that could be of interest. 


\section{Conic diffusion martingales with uniform distribu- tion}

We set out to construct a martingale diffusion process $X$ (zero drift) with marginal at time $t$ having a uniform distribution in an interval $[a(t), b(t)]$. The martingale condition implies that $\mathbb{E}\left[X_{t}\right]=\mathbb{E}\left[X_{0}\right]$ for all $t \geq 0$, whereas the uniform distribution requirement implies that $\mathbb{E}\left[X_{t}\right]=[a(t)+b(t)] / 2$ for all $t \geq 0$. Thus we have $a(t)+b(t)=a(0)+b(0)$ for all $t \geq 0$. We will assume $a(0)=b(0)=0$, taking the initial condition $X_{0}$ to be deterministic and with value zero (Dirac delta law in 0 ). Hence $b(t)=-a(t)$ for all $t \geq 0$.

With such preliminaries in mind, we state the following

Problem 1 (Designing conic martingale diffusions with given uniform law). Consider the diffusion process

$$
d X_{t}=\sigma\left(X_{t}, t\right) d W_{t}, \quad X_{0}=0 .
$$

Find a diffusion coefficient $\sigma(x, t)$ such that

1. The SDE (1) has a unique strong solution;

2. The solution of (1) at time $t>0$ is uniformly distributed in $[-b(t), b(t)]$ for a nonnegative strictly increasing continuous function $t \mapsto b(t)$ with $b(0)=0$.

In other terms, our aim is to build a diffusion martingale $X$ as in (1) such that the process $X$ has a density $p(x, t)$ at time $t>0$ at the point $x$ given by the uniform density

$$
p(x, t)=\rho(x, t):=\mathbb{1}_{\{x \in[-b(t), b(t)]\}} /(2 b(t)) .
$$

In Problem $1, b$ is restricted to be strictly increasing in time. The reason is that the tight upper (resp. lower) bound $b(t)$ (resp. $a(t)$ ) of any bounded martingale must be a non-decreasing (resp. non-increasing) function ([12]). Hence, $X$ is a conic martingale; it is a martingale that exhibits a conic behaviour. We will need strict monotonicity in the following derivation, so we assumed $b$ to be strictly increasing in Problem 1.

To derive a candidate $\sigma$ in (1) we write the forward Kolmogorov (or Fokker Planck) equation for the density $p$ of (1), impose $\rho$ to be a solution and derive the resulting $\sigma$. Once this is done, we show that the resulting SDE (1) has a unique strong solution, and confirm via moments analysis that the density is indeed uniform.

\section{Deriving the candidate SDE for a uniformly dis- tributed martingale}

The forward Kolmogorov eq. for (1) with $\rho$ plugged in as a solution reads

$$
\frac{\partial \rho(x, t)}{\partial t}=\frac{1}{2} \frac{\partial^{2}}{\partial x^{2}}\left(\sigma(x, t)^{2} \rho(x, t)\right), \quad \rho(x, 0)=\delta_{0}(x) .
$$


Now we integrate twice both sides of (3) with respect to $x$ and assume we can switch integration with respect to $x$ and differentiation with respect to $t$ (one can check a posteriori that the solution we find has a continuous partial derivative with respect to $t$ so that Leibniz's rule can be used). We obtain

$$
\frac{\partial}{\partial t}\left(\int_{-\infty}^{x}\left(\int_{-\infty}^{y} \rho(z, t) d z\right) d y\right)=\frac{1}{2} \sigma(x, t)^{2} \rho(x, t),
$$

assuming the relevant first and second derivatives with respect to $x$ on the right hand side vanish fast enough at minus infinity. Compute, substituting from (2),

$$
\varphi(x, t):=\int_{-\infty}^{x}\left(\int_{-\infty}^{y} \rho(z, t) d z\right) d y= \begin{cases}0, & \text { if } x<-b(t) \\ \frac{(x+b(t))^{2}}{4 b(t)}, & \text { if } x \in[-b(t), b(t)] \\ x, & \text { if } x>b(t)\end{cases}
$$

and note that $\varphi$ is continuous in $x$. We can write this more succinctly as

$$
\varphi(x, t)=\frac{(x+b(t))^{2}}{4 b(t)} \mathbb{I}_{\{x \in[-b(t), b(t)]\}}+x \mathbb{I}_{\{x>b(t)\}} .
$$

Thus, rewriting (4) as

$$
\frac{\partial \varphi(x, t)}{\partial t}=\frac{1}{2} \sigma(x, t)^{2} \rho(x, t),
$$

and substituting (5) we are done. To do this, we need to differentiate $\varphi$ with respect to time. The calculations are all standard but one has to pay attention when differentiating terms in (5) such as

$$
\mathbb{1}_{\{x \in[-b(t), b(t)]\}}=\mathbb{1}_{\{x \geq-b(t)\}}-\mathbb{1}_{\{x>b(t)\}}
$$

which can be differentiated in the sense of distributions. Let us show how to differentiate

$$
\frac{d}{d t} \mathbb{I}_{\{x>b(t)\}}=\frac{d}{d t} \mathbb{I}_{\left\{t<b^{-1}(x)\right\}}=-\delta_{b^{-1}(x)}(t)
$$

where the index in $\delta$ denotes the point where the Dirac delta distribution is centered. One can check that all terms involving $\delta$ 's either offset each other or are multiplied by a function that vanishes at the point of evaluation.

In the end one gets, omitting time arguments and denoting differentiation with respect to time with a dot:

$$
\frac{\partial \varphi(x, t)}{\partial t}=-\frac{-\dot{b}(2 b)(x+b)+(2 \dot{b})(x+b)^{2}}{2(2 b)^{2}} \mathbb{I}_{\{x \in[-b, b]\}} .
$$

We notice that $\dot{b}$ appears only in ratios $\dot{b} / b$, so that this quantity may be extended to time $t=0$ by continuity if needed. 
The above quantity is the left hand side of (6). We can substitute $\rho$ on the right hand side and we have that

$$
-\frac{-\dot{b}(2 b)(x+b)+(2 \dot{b})(x+b)^{2}}{2(2 b)^{2}} \mathbb{1}_{\{x \in(-b, b)\}}=\frac{1}{2} \frac{\sigma(x, t)^{2}}{2 b} \mathbb{I}_{\{x \in[-b, b]\}} .
$$

After some algebra, one obtains

$$
\sigma^{2}(x, t)=\mathbb{1}_{\{x \in[-b(t), b(t)]\}} \frac{\dot{b}(t)}{b(t)}\left(b(t)^{2}-x^{2}\right) .
$$

From the above development, we expect the diffusion coefficient $\sigma(x, t)$ defined as

$$
\sigma(x, t):=\mathbb{I}_{\{x \in[-b(t), b(t)]\}} \sqrt{\frac{\dot{b}(t)}{b(t)}\left(b(t)^{2}-x^{2}\right)} .
$$

to be a valid candidate for the solution $X$ of $(1)$ to be a martingale with uniform $[-b, b]$ law. In order to rigorously show that, we prove in the next section that SDE (1) with diffusion coefficient (7) admits a unique strong solution and that this solution has indeed a uniform law at all times.

\section{Analysis of the candidate SDE: solutions and dis- tributions}

Theorem 1 (Existence and Uniqueness of Solution for candidate SDE solving Problem 1). Let $b$ a strictly increasing function defined on $[0, T]$ and of class $C^{1}$ in $(0, T]$, with $b(0)=0$ and $T$ a positive real number. Assume the derivative of $b(t)^{2}$, namely $2 b(t) \dot{b}(t)$, to be bounded in $(0, T]$ and to have a finite limit when $t \downarrow 0$. The stochastic differential equation

$$
d X_{t}=\left(\mathbb{I}_{\left\{X_{t} \in(-b(t), b(t))\right\}} \frac{\dot{b}(t)}{b(t)}\left(b(t)^{2}-X_{t}^{2}\right)\right)^{1 / 2} d W_{t}, \quad X_{0}=0,
$$

whose diffusion coefficient is extended to $t=0$ by continuity in case the diffusion coefficient is not defined at $t=0$, admits a unique strong solution and its solution $X$ is distributed at every point in time as a uniform distribution concentrated in $(-b(t), b(t))$. We thus have a conic diffusion martingale with the cone expansion controlled by the time function $b(t)$.

Proof. By continuity of diffusion paths, the solution $X$ to the SDE (8), if it exists, belongs to $[-b(t), b(t)]$ almost surely. Indeed, the diffusion coefficient $\sigma(t, x)$ vanishes at the boundaries $\{-b(t), b(t)\}$. Because $b(t)$ is increasing, the process cannot exit the cone $[-b(t), b(t)]$. This will be further confirmed by our local time calculation in Theorem 4 for the specific case $b(t)=k t$. 
It remains to prove that the solution $X$ to (8) exists and is unique. To that end, it is enough to show that $\sigma(x, t)$ satisfies the linear growth bound and is Holder- $1 / 2$ for all $t \in[0, T][10]$.

Clearly, $\sigma(x, t)$ in $(7)$ satisfies the linear growth bound since it is uniformly bounded on $[0, T]$. To see this, notice that

$$
\sigma^{2}(x, t)=\mathbb{I}_{\{-b(t) \leq x \leq b(t)\}}(\dot{b}(t) / b(t))\left(b^{2}(t)-x^{2}\right) \leq \dot{b}(t) b(t)=\dot{b^{2}}(t) / 2
$$

and that $\dot{b}(t) b(t)$ is bounded on $(0, T]$ by assumption. Since $\sigma(x, t)$ is continuous and bounded on $(0, T]$, it admits a continuous extension at zero which is unique and bounded on $[0, T]$, showing that the extended $\sigma(x, t)$ is uniformly bounded on $[0, T]$.

We now proceed with the Holder continuity of $\sigma$. Recall that a function $f(x)$ is Holder$1 / 2$ if there exists $K \in \mathbb{R}$ such that

$$
|f(x)-f(y)| \leq K \sqrt{|x-y|}
$$

for all $x, y$ in $\operatorname{dom}(f)$. Of course, $f(x)=\sqrt{|x|}$ is Holder-1/2 on $\mathbb{R}$ since $\mid \sqrt{|x|}-\sqrt{|y|} \leq$ $\sqrt{|x-y|}$ for all $x, y$. We now check that $\sigma(t, x)$ is Holder-1/2 for all $t$ (we adopt the same strategy as in [13]). To that end, it is enough to check the Holder- $1 / 2$ continuity of

$$
\eta(t, x):=\mathbb{1}_{\{-b(t) \leq x \leq b(t)\}} \sqrt{b^{2}(t)-x^{2}}, \quad t \leq T
$$

Define $I(t):=[-b(t), b(t)]$. We check the possible cases.

1. If $x, y \notin I(t)$, the diffusion coefficient vanishes and one gets $|\eta(t, x)-\eta(t, y)|=0$

2. If $x, y \in I(t)$, using the Holder-1/2 continuity of $\sqrt{|x|}$ :

$$
\begin{aligned}
|\eta(t, x)-\eta(t, y)| & =\mid \sqrt{b^{2}(t)-x^{2}}-\sqrt{b^{2}(t)-y^{2} \mid} \\
& \leq \sqrt{\left|\left(b^{2}(t)-x^{2}\right)-\left(b^{2}(t)-y^{2}\right)\right|} \\
& =\sqrt{\left|y^{2}-x^{2}\right|} \leq \sqrt{|y+x|} \sqrt{|y-x|} \leq \sqrt{2 b(T)} \sqrt{|x-y|}
\end{aligned}
$$

3. If $x \in I(t), y>b(t)$ :

$$
|\eta(t, x)-\eta(t, y)|=|\eta(t, x)|=\sqrt{b^{2}(t)-x^{2}}=\sqrt{b(t)+x} \sqrt{b(t)-x} \leq \sqrt{2 b(T)} \sqrt{|x-y|}
$$

4. If $x \in I(t), y<-b(t)$ (so that $-y>b(t))$ :

$$
\begin{aligned}
|\eta(t, x)-\eta(t, y)| & =|\eta(t, x)| \leq \sqrt{b(t)-x} \sqrt{b(t)+x} \leq \sqrt{2 b(T)} \sqrt{x+b(t)} \\
& \leq \sqrt{2 b(T)} \sqrt{x-y}
\end{aligned}
$$

5. The case $x \notin I(t), y \in I(t)$ is similar to steps 3 and 4 . 
Hence, the solution $X$ to (8) exists and is unique. Because it is bounded and evolves between $-b(t)$ and $b(t)$, it is a conic $[-b(t), b(t)]$-martingale.

We have proven that the SDE (8) has a unique strong solution. The SDE itself has been obtained by inverting the Kolmogorov equation for a uniform marginal density at time $t$ in $[-b(t), b(t)]$, so we expect the density of the solution to be that uniform distribution. However, we haven't proven that the forward Kolmogorov equation for the density of (8) has a unique solution. To prove that our SDE (8) has the desired uniform distribution, we resort to a characterization of the uniform distribution by its moments, showing a theorem where we state that the moments of the solution of (8) are the same as the moments of the desired uniform law, and showing that this characterizes the uniform law. We start with the following

Definition 1. A probability measure $\mu$ is determined by its moments when it is the unique probability measure having this set of moments.

Lemma 1. The continuous uniform distribution on $[a, b],-\infty<a<b<\infty$, is determined by its moments.

Proof. Let us note $\alpha_{k}(p):=\int_{-\infty}^{\infty} x^{k} p(x) d x$ the $k$-th moment associated to a probability density function $p$. From Theorem 30.1 of [1], it is known that if all the moments $\alpha_{1}(p), \alpha_{2}(p), \ldots$ are finite and are such that the series

$$
S_{r}(p):=\sum_{k=1}^{\infty} \frac{\alpha_{k}(p) r^{k}}{k !}
$$

admits a positive radius of convergence, then $p$ is determined by its moments.

One concludes from this theorem that if a random variable $X$ satisfies $\mathbb{E}\left(X^{k}\right)=\alpha_{k}(p)$ for all $k \in \mathbb{N}$, then $X \sim p$ provided that (i) $\left|\alpha_{k}(p)\right|<\infty$ for $k \in \mathbb{N}$ and (ii) there exists $r>0$ such that the series $S_{r}(p)$ converges.

In particular, if the uniform density in $[a, b], \rho(x):=\frac{1}{b-a} \mathbb{1}_{\{a \leq x \leq b\}}$, satisfies (i) and (ii), then any random variable $X$ satisfying $\mathbb{E}\left(X^{k}\right)=\alpha_{k}(\rho)$ for all $k \in\{1,2, \ldots\}$ is uniformly distributed on $[a, b]$.

Let us show that (i) and (ii) are satisfied for the uniform density in $[a, b]$. Condition (i) is clearly met since the moments of the uniform distribution are finite. In particular, defining $c:=|a| \vee|b|$ one has $\left|\alpha_{k}(\rho)\right| \leq c^{k}<\infty$. On the other hand, for $r>0$,

$$
0 \leq \frac{\left|\alpha_{k}(\rho)\right| r^{k}}{k !} \leq \frac{(c r)^{k}}{k !}
$$

Since the series

$$
S_{r}^{\prime}:=\sum_{k=1}^{\infty} \frac{(c r)^{k}}{k !}
$$


converges to $e^{c r}-1$, the series $S_{r}(\rho)$ converges, too. This shows that both conditions (i) and (ii) are met for $p=\rho$, and completes the proof.

Theorem 2. The solution $X$ to the $S D E(8)$ is a uniform martingale on $[-b(t), b(t)]$ in the sense that for all $t>0, X_{t}$ is uniformly distributed on $[-b(t), b(t)]$.

Proof. From the above results, it is enough to show that all moments of the random variable $X_{t}(t>0)$ associated to eq. (8) coincide with those of the density $\mathbb{1}_{\{-b(t) \leq x \leq b(t)\}} \frac{1}{2 b(t)}$.

Let $X$ be a random variable uniformly distributed on $[a, b]$. Then,

$$
\mathbb{E}\left(X^{n}\right)=\frac{1}{n+1} \sum_{i=0}^{n}(a)^{i}(b)^{n-i} .
$$

In the special case where $a=-b$, this expression reduces to

$$
\mathbb{E}\left(X^{n}\right)= \begin{cases}\frac{b^{n}}{n+1} & \text { if } n \text { is odd } \\ 0 & \text { otherwise }\end{cases}
$$

Let us now compute the moments of

$$
X_{t}=\int_{0}^{t} \sigma\left(X_{s}, s\right) d W_{s}, \quad \sigma(t, x)=\mathbb{I}_{\{-b(t) \leq x \leq b(t)\}} \sqrt{\frac{\dot{b}(t)}{b(t)}} \sqrt{b^{2}(t)-x^{2}}
$$

solving eq. (8). By Itô's lemma:

$$
X_{t}^{n}=n \int_{0}^{t} X_{s}^{n-1} d X_{s}+\frac{1}{2} n(n-1) \int_{0}^{t} X_{s}^{n-2} \sigma^{2}\left(X_{s}, s\right) d s
$$

and we can compute the expression for the $n$-th moment, $n \geq 2$ using a recursion. Using the property that Itô 's integrals have zero expectation and exchanging integration and expectation operators, which is possible since $X_{s}^{n-2} \sigma^{2}\left(X_{s}, s\right)$ is bounded for all $s$ and $n \geq 2$, we obtain

$$
\begin{aligned}
\mathbb{E}\left(X_{t}^{n}\right) & =n \mathbb{E}\left(\int_{0}^{t} X_{s}^{n-1} d X_{s}\right)+\frac{1}{2} n(n-1) \mathbb{E}\left(\int_{0}^{t} X_{s}^{n-2} \sigma^{2}\left(X_{s}, s\right) d s\right) \\
& =\frac{n(n-1)}{2}\left(\int_{0}^{t} b(s) \dot{b}(s) \mathbb{E}\left(X_{s}^{n-2}\right) d s-\int_{0}^{t} \frac{\dot{b}(s)}{b(s)} \mathbb{E}\left(X_{s}^{n}\right) d s\right)
\end{aligned}
$$

Notice that we have postulated in the last equality that the indicator $\mathbb{1}_{\left\{-b(s) \leq X_{s} \leq b(s)\right\}}$ in $\sigma(t, x)$ is always 1 . This is a natural assumption: it says that $X$ cannot stay on a boundary with a strict positive probability for a given period of time. This happens because in case $X$ reaches $\pm b(t)$ at some time $t$, the process is locally frozen $(\sigma(t, x)=0)$ but the boundary $b(t)$ keeps on growing (see also the local time calculation in Theorem 4 for the specific case $b(t)=k t)$. 
Obviously, $\mathbb{E}\left(X_{t}\right)=X_{0}=0$ since $X$ is a martingale and one concludes from eq. (9) that the $n$-th moment of $X_{t}$ is zero when $n$ odd. For $n$ even, eq. (9) can be written as

$$
f(t, n)=\frac{n(n-1)}{2}\left(\int_{0}^{t} b(s) \dot{b}(s) f(s, n-2) d s-\int_{0}^{t} \frac{\dot{b}(s)}{b(s)} f(s, n) d s\right)
$$

with $f(t, n):=\mathbb{E}\left(X_{t}^{n}\right)$. This can be written as a recursive differential equation

$$
\frac{\partial f(t, n)}{\partial t}=\dot{b}(t) \frac{n(n-1)}{2}\left(b(t) f(t, n-2)-\frac{1}{b(t)} f(t, n)\right)
$$

with the constraint that $f(t, 0)=\mathbb{E}\left(X_{t}^{0}\right)=1$. The solution to this equation is $f(t, n)=$ $b^{n}(t) /(n+1)$. One concludes that $X_{t}$ is uniform on $[-b(t), b(t)]$ since all the odd moments are zero and all the even moments are given by

$$
\mathbb{E}\left(X_{t}^{n}\right)=\frac{b^{n}(t)}{n+1}
$$

and agree with those of a random variable uniformly distributed on $[-b(t), b(t)]$.

Remark 1 (Peacocks). It is important to notice that our process $(1)$ with general $b(t)$ leads to a construction of martingales associated to the peacock $b(t) U$ where $U$ is uniform on $[-1,1]$ (peacocks are discussed in general in $[9]$ ).

The special case $b(t)=k t$ gives us a conic martingale with uniform distribution where the boundaries grow symmetrically and linearly in time. This example was considered originally in [3]. Another interesting case is $b(t)=k \sqrt{t}$. We will discuss such cases in the following sections and relate them to specific peacock processes literature [9].

\section{Mean reverting uniform diffusions with constant boundaries}

Consider the SDE (8) and define

$$
Z_{t}=X_{t} / b(t), \quad X_{t}=b(t) Z_{t}
$$

Since $X_{t}$ is uniform in $(-b(t), b(t)), Z_{t}$ will be uniform in $[-1,1]$ for $t>0$. We extend the distribution to a uniform $[-1,1]$ also at time 0 . Thus, with this deterministic re-scaling, we have a process $Z$ with fixed uniform distribution and fixed boundaries. We can differentiate $Z$ to obtain its SDE. One has, via Leibnitz rule

$$
d Z_{t}=-\frac{\dot{b}(t)}{b(t)} Z_{t} d t+\left(\frac{\dot{b}(t)}{b(t)}\left(1-Z_{t}^{2}\right)\right)^{1 / 2} \mathbb{1}_{\left\{Z_{t} \in[-1,1]\right\}} d W_{t}, \quad Z_{0}=\zeta \sim U([-1,1])
$$


Here we assume the initial condition $\zeta$ to be independent of the driving Brownian motion.

If instead we aim at obtaining a standard uniform, in $[0,1]$, we adopt a slightly different transformation:

$$
Y_{t}=\left(X_{t} / b(t)+1\right) / 2=X_{t} /(2 b(t))+1 / 2
$$

from which

$$
X_{t}=2 b\left(Y_{t}-1 / 2\right) .
$$

By Leibnitz rule we have the following

Theorem 3. Consider the SDEs

$d Y_{t}=\frac{\dot{b}}{b}\left(1 / 2-Y_{t}\right) d t+\frac{1}{2 b}\left(\mathbb{I}_{\left\{Y_{t} \in(0,1)\right\}} b(t) \dot{b}(t)\left(1-4\left(Y_{t}-1 / 2\right)^{2}\right)\right)^{1 / 2} d W_{t}, \quad Y_{0}=\xi \sim U([0,1])$

and

$$
d Z_{t}=-\frac{\dot{b}(t)}{b(t)} Z_{t} d t+\left(\frac{\dot{b}(t)}{b(t)}\left(1-Z_{t}^{2}\right)\right)^{1 / 2} \mathbb{1}_{\left\{Z_{t} \in(-1,1)\right\}} d W_{t}, \quad Z_{0}=\zeta \sim U([-1,1])
$$

with $\xi$ and $\zeta$ independent of $W$. The solutions of these SDEs mean-revert to $1 / 2$ and 0 respectively with speed $\dot{b} / b$ and are distributed at any point in time as a standard uniform random variable and as a uniform $[-1,1]$ random variable respectively.

$Y$ can be used for example to model the dynamics of recovery rates or probabilities in the case of no information (maximum entropy), whereas $Z$ can be used as a model for stochastic correlation.

\section{Specific choices of the boundary $b(t)$ and links with peacocks}

In this section we present a number of different qualitative choices for $b(t)$.

\subsection{The sub-linear square-root case $b(t)=\sqrt{t}$}

The case $b(t)=\sqrt{t}$ for $(8)$, which leads to

$$
d X_{t}=\frac{1}{\sqrt{2}} \sqrt{1-\frac{X_{t}^{2}}{t}} \mathbb{I}_{\left\{X_{t} \in[-\sqrt{t}, \sqrt{t}]\right\}} d W_{t}, \quad X_{0}=0
$$

corresponds exactly to the solution presented in [9], where the authors establish in formula (6.60) that the SDE

$$
d X_{t}=\frac{1}{\sqrt{2}} \sqrt{1-\frac{X_{t}^{2}}{t}} d W_{t}
$$


has a solution which is a continuous martingale such that $X_{t}$ has the same law as $\sqrt{t} U, U$ being a r.v. uniformly distributed on $[-1,+1]$. To do so, they study, for any $s \in \mathbb{R}$ (not necessarily positive) the SDE, for $s \leq t$

$$
Y_{t}=Y_{s}+\int_{s}^{t} \frac{1}{\sqrt{2}} \sqrt{1-Y_{u}^{2}} d W_{u}-\frac{1}{2} \int_{s}^{t} Y_{u} d u
$$

(which has a unique strong solution) and then show that $X_{t}=\sqrt{t} Y_{\log t}, X_{0}=0$ is a solution. We arrived to the same equation via the more general setting in $b(t)$ by inverting the Kolmogorov forward equation. Clearly, this $b(t)$ fits the assumption of Theorem 1 since the derivative of $b^{2}(t)=t$ is 1 and is thus bounded on $[0, \infty]$.

\subsection{The linear case $b(t)=k t$ : numerical examples, boundary be- haviour and activity}

Again, $b(t)=t$ fits the assumption of Theorem 1 since the derivative of $b^{2}(t)$ is bounded on $[0, T]$ for any $T \in \mathbb{R}^{+}$. Our previous SDEs for $X(8)$ and $Z$ (10) specialize to

$$
d X_{t}=\mathbb{I}_{\left\{X_{t} \in[-k t, k t]\right\}} \frac{1}{\sqrt{t}} \sqrt{(k t)^{2}-X_{t}^{2}} d W_{t}, \quad X_{0}=0, \quad X_{t} \sim U([-k t, k t]) \text { for all } t>0
$$

and

$$
d Z_{t}=-\frac{1}{t} Z_{t} d t+\mathbb{I}_{\left\{Z_{t} \in[-1,1]\right\}} \frac{1}{\sqrt{t}} \sqrt{1-Z_{t}^{2}} d W_{t}, Z_{0} \sim Z_{t} \sim U(-1,1) \text { for all } t>0 .
$$

As a numerical example we implement the Euler scheme for $X$. We know from [8] that under our assumptions the Euler scheme converges in probability. We thus implement a Euler scheme for the SDE for $X$ and then plot a histogram of the density. This is shown in Figure 1. Moreover, we show in Figure 2 a few sample paths of the process $X$.

We now study the behaviour of the process $X$ solving (11) near the conic boundary. To do so, we may consider the re-scaled process $Z$. The boundary behaviour of $X$ at $-k t$ and $k t$ will be the same as the behaviour of $Z$ at -1 and 1 . In particular, $X$ will not spend time at the boundaries $-k t$ and $k t$ if and only if $Z$ will not spend time at -1 and 1 .

We can use local time as an investigative tool for the behaviour at the boundary.

Theorem 4 (Local time for the solution of the SDE (12) for $Z_{t}=X_{t} / k t$ with $X_{t}$ in (11)). The local time for $Z$ at points -1 and 1 is zero.

Proof. Using the fact that $d\langle Z\rangle_{s}=\frac{1-Z_{s}^{2}}{s} d s$, one has, for $t>\epsilon>0$,

$$
\begin{aligned}
t & \geq \int_{0}^{t} \mathbb{I}_{\left\{Z_{s}>-1\right\}} d s=\int_{0}^{t} \mathbb{I}_{\left\{Z_{s}>-1\right\}} \frac{s}{1-Z_{s}^{2}} d\langle Z\rangle_{s} \geq \int_{\epsilon}^{t} \mathbb{I}_{\left\{Z_{s}>-1\right\}} \frac{s}{1-Z_{s}^{2}} d\langle Z\rangle_{s} \\
& \geq \epsilon \int_{\epsilon}^{t} \mathbb{I}_{\left\{Z_{s}>-1\right\}} \frac{1}{1-Z_{s}^{2}} d\langle Z\rangle_{s}
\end{aligned}
$$



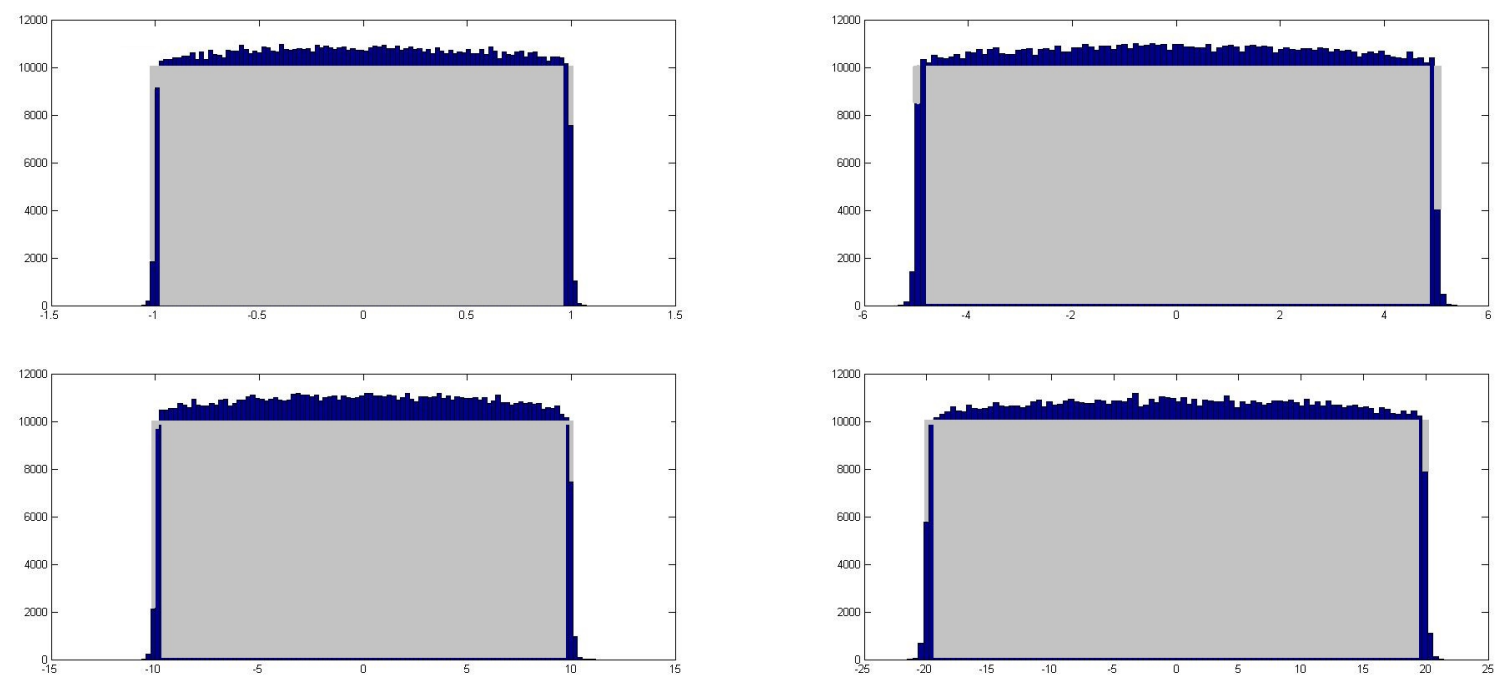

Figure 1: Histograms with 100 bins for the density of the SDE (11) at time $t$ with $k=1$ for 1 Million scenarios via Euler scheme: top left $t=1 y$, top right $5 y$, bottom left $10 y$ and bottom right 20y. Time step is 0.1 years. Simulation histograms in blue, theoretical distribution in gray.

From the occupation time formula [11, Cor. 1.6, Chapter VI]

$$
\int_{0}^{t} \mathbb{I}_{\left\{Z_{s}>-1\right\}} \frac{1}{1-Z_{s}^{2}} d\langle Z\rangle_{s}=\int_{-1}^{\infty} L_{t}^{z} \frac{1}{1-z^{2}} d z
$$

it follows that

$$
t \geq \int_{-1}^{\infty}\left(L_{t}^{z}-L_{\epsilon}^{z}\right) \frac{1}{1-z^{2}} d z
$$

where $L^{z}$ is the local time of $Z$ at level $z$. The quantity $\left(L_{t}^{z}-L_{\epsilon}^{z}\right)$ is non negative and the integral on the right hand side converges, hence $\left(L_{t}^{(-1)}-L_{\epsilon}^{(-1)}\right)=0$. The local time being continuous, $L_{\epsilon}^{(-1)} \rightarrow 0$ when $\epsilon$ goes to 0 , and it follows that $L_{t}^{(-1)}=0$. By spatial symmetry, we have the same result for the point +1 .

More qualitatively, we observe that $Z$ in (12) mean-reverts to 0 with speed $1 / t$. The speed will be very large for small time but will become almost zero when time is large. The diffusion coefficient, similarly, is divided by $\sqrt{t}$, so it will tend to vanish for large $t$. This is confirmed by the following activity calculation. We may conclude that the process will not be absorbed in the boundary and will tend to "slow down" in time, while maintaining a uniform distribution.

We show that the pathwise activity of the uniform $(-1,1)$ process $Z$ is vanishing for large $t$ in the sense that the deviation of $Z_{t+\delta}(\omega)$ from $Z_{t}(\omega)$ collapses to zero for all $\delta>0$, all $\omega \in \Omega$ as $t \rightarrow \infty$. 
D. Brigo, M. Jeanblanc \& F. Vrins. SDEs with uniformly-distributed solutions.

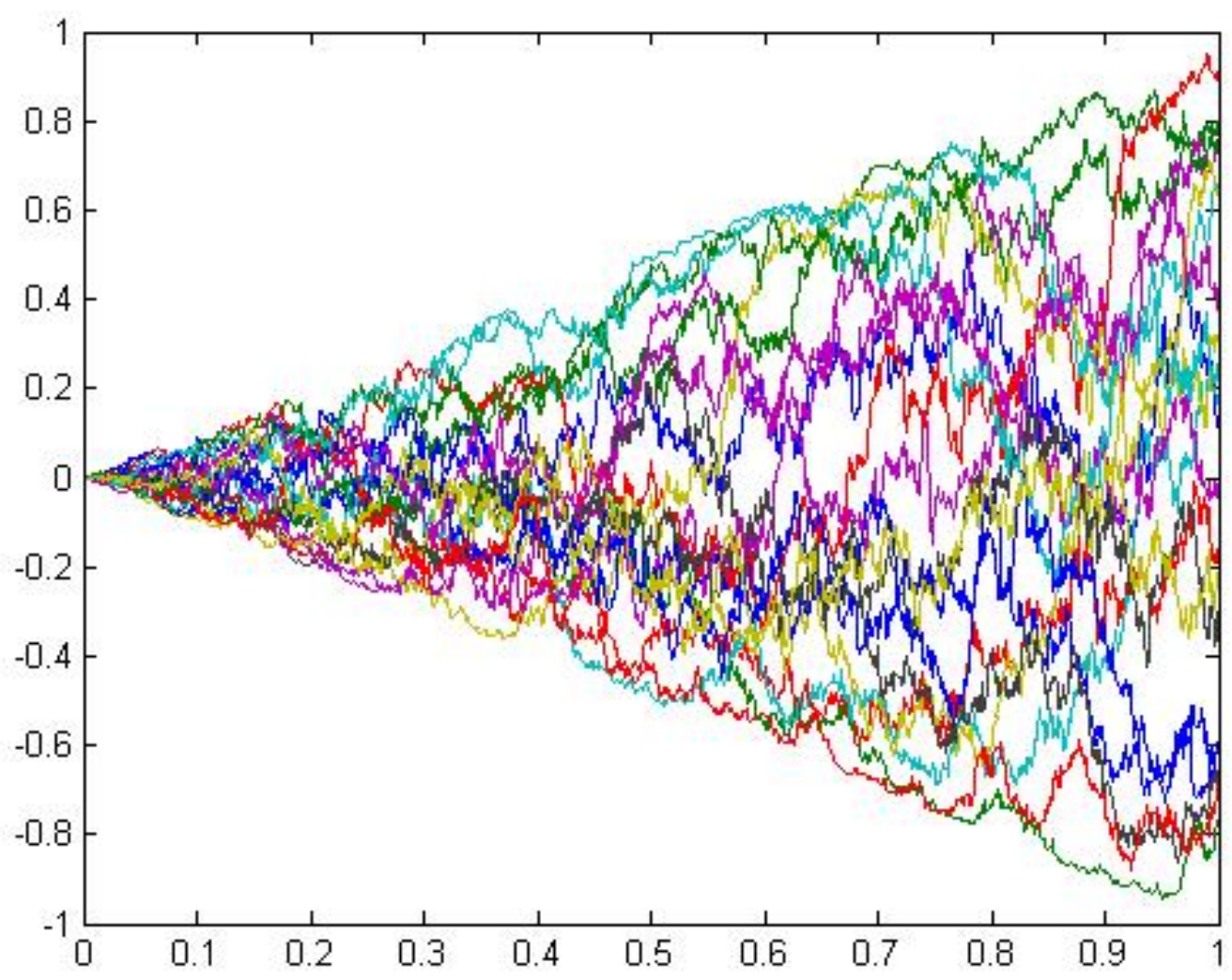

Figure 2: 20 paths of the $\operatorname{SDE}(11)$ at time $1 y$ with $k=1$. Time step is 0.01 years. Euler Scheme 


\section{Lemma 2.}

$$
\forall \delta>0, \operatorname{Var}\left(Z_{t+\delta}-Z_{t}\right) \rightarrow 0 \text { as } t \rightarrow \infty .
$$

Proof. Notice that for all $t>0, \mathbb{E}\left(Z_{t}\right)=0$ so that $\operatorname{Var}\left(Z_{t}\right)=\mathbb{E}\left(Z_{t}^{2}\right)=\sigma^{2}$ where $\sigma^{2}=$ $\sqrt{2} / 12$ is the variance of a zero-mean uniform random variable distributed on $[-1,1]$. Then,

$$
\operatorname{Var}\left(Z_{t+\delta}-Z_{t}\right)=\operatorname{Var}\left(Z_{t+\delta}^{2}\right)+\operatorname{Var}\left(Z_{t}^{2}\right)-2 \mathbb{C o v}\left(Z_{t}, Z_{t+\delta}\right)=2\left(\sigma^{2}-\mathbb{E}\left(Z_{t} Z_{t+\delta}\right)\right) .
$$

Since $Z$ is bounded, one can rely on Fubini's theorem for all $t>0$ and exchange time-integration and expectation:

$$
\begin{aligned}
\mathbb{E}\left(Z_{t} Z_{t+\delta}\right) & =\mathbb{E}\left(Z_{t}\left(Z_{t}-\int_{t}^{t+\delta} \frac{Z_{s}}{s} d s+\int_{t}^{t+\delta} \sigma\left(s, Z_{s}\right) d W_{s}\right)\right) \\
& \left.=\mathbb{E}\left(Z_{t}^{2}\right)-\mathbb{E}\left(Z_{t} \int_{t}^{t+\delta} \frac{Z_{s}}{s} d s\right)+\mathbb{E}\left(\int_{t}^{t+\delta} Z_{t} \sigma\left(s, Z_{s}\right) d W_{s}\right)\right) \\
& =\sigma^{2}-\int_{t}^{t+\delta} \frac{\mathbb{E}\left(Z_{t} Z_{s}\right)}{s} d s
\end{aligned}
$$

Observing that $\mathbb{E}\left(Z_{s} Z_{t}\right)=\sqrt{\operatorname{Var}\left(Z_{t}\right) \operatorname{Var}\left(Z_{s}\right)} \rho(s, t)=\sigma^{2} \rho(s, t)$ where $\rho(s, t)$ is the correlation between $Z_{t}$ and $Z_{s}$, one gets

$$
\operatorname{Var}\left(Z_{t+\delta}-Z_{t}\right)=2 \sigma^{2} \int_{t}^{t+\delta} \frac{\rho(s, t)}{s} d s \leq 2 \sigma^{2} \int_{t}^{t+\delta} \frac{1}{s} d s=2 \sigma^{2} \delta \frac{\delta+2 t}{t(t+\delta)^{2}} .
$$

The RHS of the above expression tends to zero as $t \rightarrow \infty$ showing that the variance of increments of $Z$ over a time interval of fixed length $\delta$ collapses to zero as $t \rightarrow \infty$.

The activity result can be generalized to the following

Lemma 3. Let $X_{t}=x_{0}+\int_{0}^{t} \theta_{s} d W_{s}$ and suppose $X=\left(X_{t}\right)_{t \geq 0}$ is a bounded non-vanishing martingale in the sense that for all $t \geq 0, a \leq X_{t} \leq b$ and $\mathbb{P}\left(\theta_{t}=0\right)<1$. Then, the path activity of $X$ is collapsing to zero as time passes.

Proof. Since martingales have independent increments, the variance of increments is the increment of the variances:

$$
\mathbb{V} a r\left(X_{t+\delta}-X_{t}\right)=\mathbb{V} \operatorname{ar}\left(X_{t+\delta}\right)+\operatorname{Var}\left(X_{t}\right)-2 \mathbb{C} \operatorname{Cov}\left(X_{t}, X_{t+\delta}\right)=\operatorname{Var}\left(X_{t+\delta}\right)-\mathbb{V} \operatorname{ar}\left(X_{t}\right) .
$$

Because the diffusion coefficient $\theta_{s}$ does not vanish on $(t, t+\delta)$,

$\operatorname{Var}\left(X_{t+\delta}-X_{t}\right)=\int_{t}^{t+\delta} \mathbb{E}\left(\theta_{s}^{2}\right) d s>0$ showing that the variance of $X_{t}$ is monotonically increasing with respect to $t$. But the variance of a bounded process is bounded. In particular, it is easy to see that $\operatorname{Var}\left(X_{t}\right) \leq\left(x_{0}-a\right)\left(b-x_{0}\right)$ since $\mathbb{E}\left(X_{t}\right)=x_{0}$ and the variance of any random variable $Y$ with expectation $\mu_{Y}$ and taking values in $[a, b]$ is bounded from above by the variance of $a+(b-a) B$ where $B$ is a Bernoulli random variable with parameter $\pi=\left(\mu_{Y}-a\right) /(b-a)$. Hence, $\operatorname{Var}\left(X_{t}\right)$ and $\operatorname{Var}\left(X_{t+\delta}\right)$ are increasing to the same limit, proving that for all $\epsilon>0$ there exists $t^{\star}$ such that $\operatorname{Var}\left(X_{t+\delta}-X_{t}\right)<\epsilon$ for all $t>t^{\star}$. 


\section{Conclusions and further research}

We introduced a way to design Stochastic Differential Equations of diffusion type admitting a unique strong solution distributed as a uniform law with conic time-boundaries. In other terms, we provided a general constructive technique to design conic diffusion martingales with given conic boundaries. While the general result is new, some such martingales where previously found in the peacock processes literature, where the case with square root of time boundaries had been dealt with. We introduced other new particular cases, such as the time-linear boundary case. We further introduced general mean-reverting diffusion processes having a constant uniform law at all times. This may be used to model random probabilities, random recovery rates or random correlations. The techniques we introduced here would also allow to design diffusion martingales whose boundary grows exponentially fast in time, or whose boundary tends to a fixed asymptotic constant interval $[\gamma, \gamma]$ as times goes to infinity. We will examine such cases, including

$$
b(t)=\beta \exp (\alpha t), \quad b(t)=\gamma-\frac{1}{t+\frac{1}{\gamma}}
$$

(for positive real constants $\alpha, \beta$ and $\gamma$ ) in future work.

\section{Acknowledgements}

The research of Monique Jeanblanc is supported by Chair Markets in Transition (Fédération Bancaire Française) and Labex ANR 11-LABX-0019.

Monique Jeanblanc thanks C. Profeta for stimulating discussions.

\section{References}

[1] Billingsley, P. (1995). Probability and measure, Wiley.

[2] Brigo, D. (1997). On nonlinear SDEs whose densities evolve in a finite-dimensional family. In: Stochastic Differential and Difference Equations, Progress in Systems and Control Theory, vol. 23, pp. 11-19, Birkhäuser, Boston.

[3] Brigo, D. (1999). On diffusion processes with uniform distributions and their Monte Carlo discretization. Banca IMI PDG Preprint.

[4] Brigo, D (2000). On SDEs with marginal laws evolving in finite-dimensional exponential families, STAT PROBABIL LETT, Vol: 49, Pages: 127 - 134

[5] D. Brigo, F. Mercurio (2000). Option Pricing Impact of Alternative Continuous-Time Dynamics for Discretely-Observed Stock Prices, Finance and Stochastics Vol. 4 N. 2, pp. $147-159$. 
D. Brigo, M. Jeanblanc \& F. Vrins. SDEs with uniformly-distributed solutions.

[6] D. Brigo, F. Mercurio (2001). Displaced and Mixture Diffusions for AnalyticallyTractable Smile Models, in: Geman, H., Madan, D.B., Pliska, S.R., Vorst, A.C.F. (Editors), Mathematical Finance - Bachelier Congress 2000, Springer.

[7] Dupire, B. (1994). Pricing with a smile, Risk (7), 18-20.

[8] I. Gyongy, N. Krylov (1996). Existence of strong solutions for Itos stochastic equations via approximations, Probability Theory Related Fields 105, 143158.

[9] Hirsch, F., Profeta, C., Roynette, B., Yor, M. (2011). Peacocks and Associated Martingales, with Explicit Constructions. Springer-Verlag, Berlin.

[10] Karatzas, I. and Shreve, S. (2005). Brownian Motion and Stochastic Calculus, Springer.

[11] Revuz, D. and Yor, M. (1999), Continuous Martingales and Brownian Motion, Springer Verlag, Third edition.

[12] Vrins, F., Conic Martingales, International Conference of the Bachelier Finance Society, June 2014, Brussels.

[13] Vrins, F., Jeanblanc, M. Conic Martingales from Stochastic Integrals, Submitted. 